\title{
Development of a low cost star tracker for the SHEFEX mission
}

\author{
Malak Samaan ${ }^{*}, 1$, Stephan Theil ${ }^{2}$ \\ DLR, German Aerospace Center, Institute of Space Systems, Bremen D-28359, Germany
}

\section{A R T I C L E I N F O}

\section{Article history:}

Received 14 December 2009

Received in revised form 28 July 2011

Accepted 29 September 2011

Available online $\mathrm{xxxx}$

\section{Keywords:}

Star tracker

Attitude determination

Re-entry

SHEFEX

Spacecraft navigation

\begin{abstract}
A B S T R A C T
German Aerospace Center (DLR) is designing and developing a novel star tracker to be used as the primary attitude sensor for the SHEFEX mission. The developed star tracker is considered to be a low cost and low accuracy sensor that is suitable for the proposed mission and the attitude accuracy requirements. This sensor is based on an off-the-shelf camera and a PC/104 computer communicating with the navigation computer. The attitude determination software consists of a processing chain including the camera control, the image processing, the star identification and the attitude estimation.

The emphasis of the work is on developing a simple but robust system. Therefore, different algorithm concepts for each element of the chain have been implemented, tested and compared. Several night sky tests and star image simulation software have been implemented and used to verify the performance of the sensor in real-time End-to-End tests. The paper presents the star tracker system, details some newly developed algorithms and analyzes the test results.
\end{abstract}

(c) 2011 Elsevier Masson SAS. All rights reserved.

\section{Introduction}

After the successfully launched hypersonic SHarp Edge Flight EXperiment (SHEFEX) in 2005, DLR has scheduled a second reentry rocket experiment SHEFEX 2 [10] for 2011. The novel flight control system based on steerable canard fins requires a precise knowledge of the inertial spacecraft attitude. Based on the rocket's flight path angle requirements, the attitude accuracy must be better than $0.17 \mathrm{deg}$. The flight path angle is determined via GPS measurements and can be delivered with the required accuracy, but the attitude delivered by the INS is subject to heavy errors due to misalignment of the gyro and drift of the attitude error due to a high vibration load.

To solve this problem, the GNC division of the DLR Institute of Space Systems is developing a hybrid navigation system [11] combining measurements from an inertial measurement unit (IMU), a GPS receiver and a star tracker. This solution has the advantage that the star tracker attitude estimates can be used to compensate the drift errors of the IMU. For this purpose, a star tracker is to be conceived and integrated in an inertial navigation system, updating the attitude information before re-entry.

The developed hybrid navigation system uses a real-time navigation computer (NC) receiving input signals from the GPS, the IMU and the star tracker. The principle is that the state vector

\footnotetext{
* Corresponding author.

E-mail addresses: Malak.Samaan@DLR.de (M. Samaan), Stephan.Theil@DLR.de (S. Theil).

1 Research Engineer, PhD, GNC Division.

2 Head of GNC Division, PhD, DLR Bremen.
}

of the rocket (consisting of the spacecraft's position, velocity, attitude and angular velocity) is propagated with the help of IMU measurements submitted to a strap down computation. The state is corrected by measurements of the GPS receiver and the star tracker. After transformations of the state vector, delivered in the Earth Centered Inertial frame, to the appropriate output frame, the navigation solution is submitted to the Canard Control Computer (CCC) at a rate of $100 \mathrm{~Hz}$.

To ensure synchronous measurements, an internal clock of the navigation computer triggers the IMU and the star tracker and also controls the state propagation. After receiving the trigger signal, the GPS delivers the position and the star tracker delivers the attitude information as a quaternion to the navigation computer.

With the choice of developing a hybrid navigation system, the GNC division also decided not to rely on a commercial star tracker as absolute attitude sensor, but to design its own one based on offthe-shelf components. This choice should also allow DLR to gain experience on star trackers development.

A robust and useful utilization of the star tracker in the system shall be ensured by the following requirements:

- Attitude measurement for the lost in space case (no a priori information);

- Attitude error less than $0.1 \mathrm{deg}$ in each of the three axes;

- Ability to deliver attitude information for angular velocities up to $2 \mathrm{deg} / \mathrm{s}$;

- Total processing time less than 100 ms, i.e. the attitude is estimated at $10 \mathrm{~Hz}$;

- Shutter time shorter than $50 \mathrm{~ms}$ due to high angular rates; 

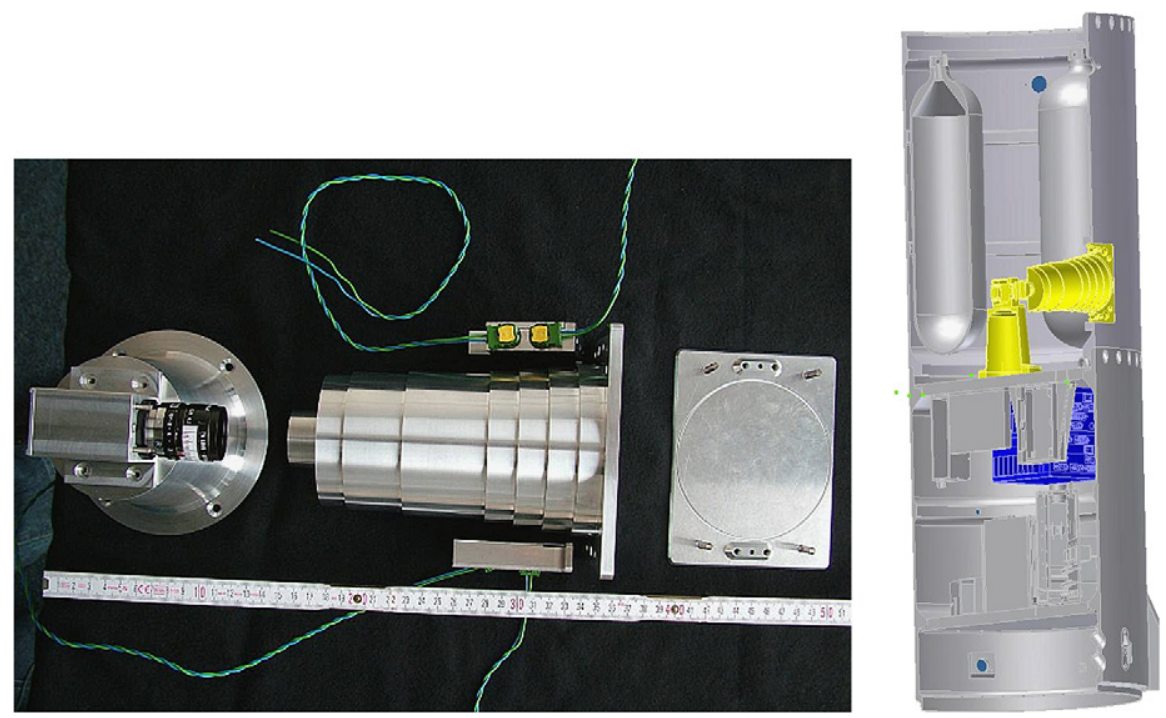

Fig. 1. The star tracker hardware and within the rocket body structure.

- Robustness regarding unfavorable situations, which happened when non-steller objects appear in the FOV.

The principle driving the development of the star tracker and especially the related software is that it is better to generate an error message than to deliver wrong attitude information. This principle is the criterion for a robust attitude estimation.

A special condition of the flight experiment is that, unlike stable pointing satellites, the angular rates of the sounding rocket are expected to be in the order of $2 \mathrm{deg} / \mathrm{s}$ during the star tracker operating time. This time frame starts shortly after the separation of the second stage and ends just before re-entry, when the rocket effectuates a spin manoeuvre at high angular velocity.

The availability of the star tracker is also an important issue. The factors which influence this availability are the exclusion angles between the star camera's optical axis and both the Sun vector and the horizon vector in the star tracker frame. Therefore, the location and the orientation of the star tracker in the rocket has to be carefully chosen. The requirements regarding these angles are assumed to be:

- Sun exclusion angle: $90 \mathrm{deg}$;

- Earth exclusion angle: $45 \mathrm{deg}$.

\section{Star tracker hardware}

The hardware used for the SHEFEX 2 star tracker includes a suitable CCD camera with a corresponding lens and a PC/104 computer [1] for the image processing chain. All equipment had to demonstrate its acceptance and qualification levels for operation in space environment. The flight hardware and the integration of the camera together with the baffle shielding the optics colored in yellow and the star tracker computer colored in blue are depicted in Fig. 1. (For interpretation of the references to color in this figure, the reader is referred to the web version of this article.)

\subsection{Star camera and optics}

For the SHEFEX 2 experiment, the goal is to select an off-theshelf camera, giving the best images at a reasonable price. With this objective, it is clear that only sensors with limited sensitivity can be chosen. This in turn leads to a reduced number of stars that can be detected when respecting a required exposure time of $50 \mathrm{~ms}$ or less. The need arises to select a lens that gives a wide
Table 1

Characteristics of PROSILICA EC655.

\begin{tabular}{ll}
\hline Weight & $84 \mathrm{~g}$ \\
Dimensions & $33 \mathrm{~mm} \times 46 \mathrm{~mm} \times 56 \mathrm{~mm}$ \\
Power consumption & $2.5 \mathrm{~W}$ \\
CCD size & $659 \times 493$ \\
Pixel size & $9.9 \mu \mathrm{m} \times 9.9 \mu \mathrm{m}$ \\
Exposure control & $10 \mu \mathrm{s}$ to $10 \mathrm{~s}$, in $1 \mu \mathrm{s}$ increment \\
Frame rate & $90 \mathrm{fps}$ at full resolution \\
Interface standard & IEEE 1394 (FireWire) \\
Lens mount & C-mount with adjustable back-focus \\
\hline
\end{tabular}

field of view, in order to be able to detect enough stars in one image. Further, the wanted limitation of image smear is responsible for the short required exposure times, while still having a maximum signal-to-noise ratio (SNR).

Based on several in-door and night sky tests, it was decided to use the monochromatic CCD camera (model: PROSILICA EC655). The relevant characteristics (taken from [6]) are listed in Table 1.

The lens used for the star tracker camera is a PENTAX $25 \mathrm{~mm}$ lens with manually adjustable focus and brightness. The field of view obtained with this lens is $14.87 \mathrm{deg} \times 11.15 \mathrm{deg}$, which results in a diagonal FOV of $18.57 \mathrm{deg}$.

The last component of the optical system is the baffle, a standard in star tracker hardware. The shape of the baffle results from the conditions for the exclusion angles. The baffle as it was designed for the SHEFEX 2 mission is protecting the camera from light rays that have an angle of more than 50 degrees with the optical axis.

\subsection{Star tracker computer}

The star tracker computer employed for the SHEFEX 2 mission is a PC/104 model, that has already been flown in previous experiments. To make it completely safe against vibration and vacuum, some connectors were changed and glued and some capacitors were replaced. The computer must be able to communicate with the NC, the camera and the power supply box.

This computer is composed of three modules, that are set one on top of the other. The first one is the power module, supplied with $28 \mathrm{~V}$ from the central power supply. The second module is the CPU-module with $650 \mathrm{MHz}$ speed, including all the main components such as memory and flash drive. It also gives the possibility to use USB and COM ports for communication with external devices. The last module is the FireWire (IEEE 1394) module 


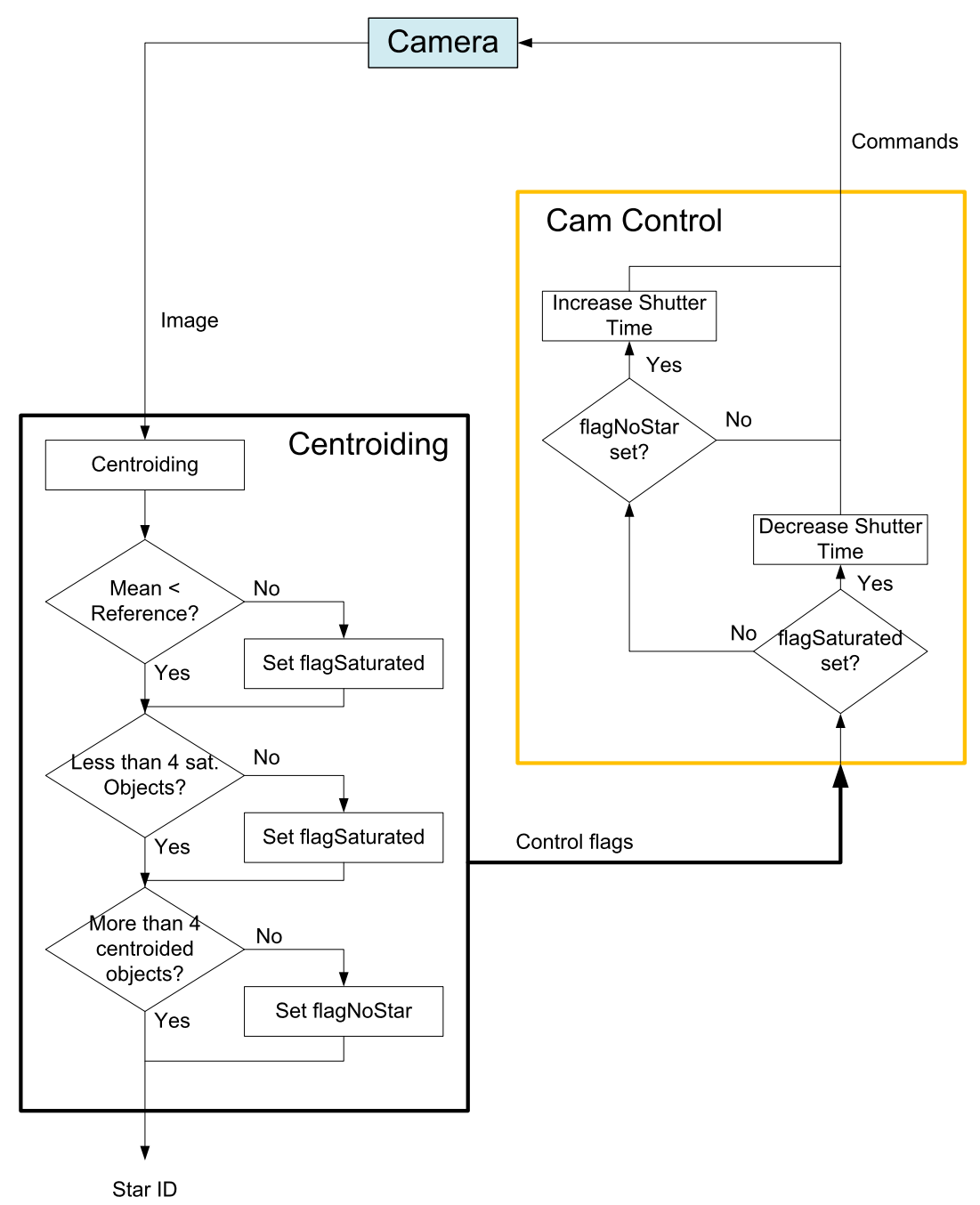

Fig. 2. Flow chart of the camera control mechanism.

needed for the camera interface. The fact that the $\mathrm{NC}$ is also a $\mathrm{PC} / 104$ and has the same shape makes it possible to combine both computers in one stack.

\section{Algorithms development}

The image processing chain used for attitude estimation based on the star images consists of the camera control, the image processing, the star identification and the attitude estimation. Each selected algorithm will be described shortly with emphasis on innovative parts and algorithm tests.

\subsection{Camera exposure time control}

The main task for the camera control is to adjust the camera parameters during the operation time. From the many parameters that can be controlled via the camera interface, only the exposure time and the camera gain is needed in our star tracker application.

According to the specifications of the PROSILICA EC655 the exposure time can vary between $10 \mu \mathrm{s}$ and $1 \mathrm{~s}$. However the onboard exposure time limit has to be chosen according to the results of many night sky tests. The upper limit is fixed by the intended processing rate of the pictures and the image smear. If the required processing rate is $10 \mathrm{~Hz}$, so the integration time plus the processing time must be below $100 \mathrm{~ms}$. To reduce the effect of image smear, the upper limit of the exposure time will be set to $50 \mathrm{~ms}$, resulting in a maximum image smear of 4.5 pixel.

As stated above, the upper limit is also selected with the aid of night sky tests. During these tests, the processing could be done in most cases with an integration time of $50 \mathrm{~ms}$ or less. Knowing that the atmosphere absorbs about 30 percent of incident light in the sensitivity band of the CCD, the limit of $50 \mathrm{~ms}$ on ground would be equivalent to about $35 \mathrm{~ms}$ in space. Therefore, a certain margin of the integration time for the difference between the ground tests and the real on-orbit operations should be taken into account.

The control of the exposure time can only be done with the help of some Image Processing criteria describing the image quality. These criteria can be determined during the star centroiding. The first criterion will be determined using the averaging of the images grayscale values. This mean value is compared to a reference; if it exceeds the reference, a flag called flagSaturated, will be set. The main purpose of this comparison is to avoid augmenting the exposure time when a very bright object is in the FOV or some object is reflecting light into the optics. In practice, this operation should only be positive in case of some unexpected image, that doesn't entirely represent a star sky. The camera control mechanism is shown as a flow chart in Fig. 2.

The same flag, flagSaturated will also be set in the case of too many saturated pixels (for 8 bit digitization: pixels with a grayscale value) in the detected stellar objects. It is not convenient to use the same method as described in the last paragraph for this check, nor 


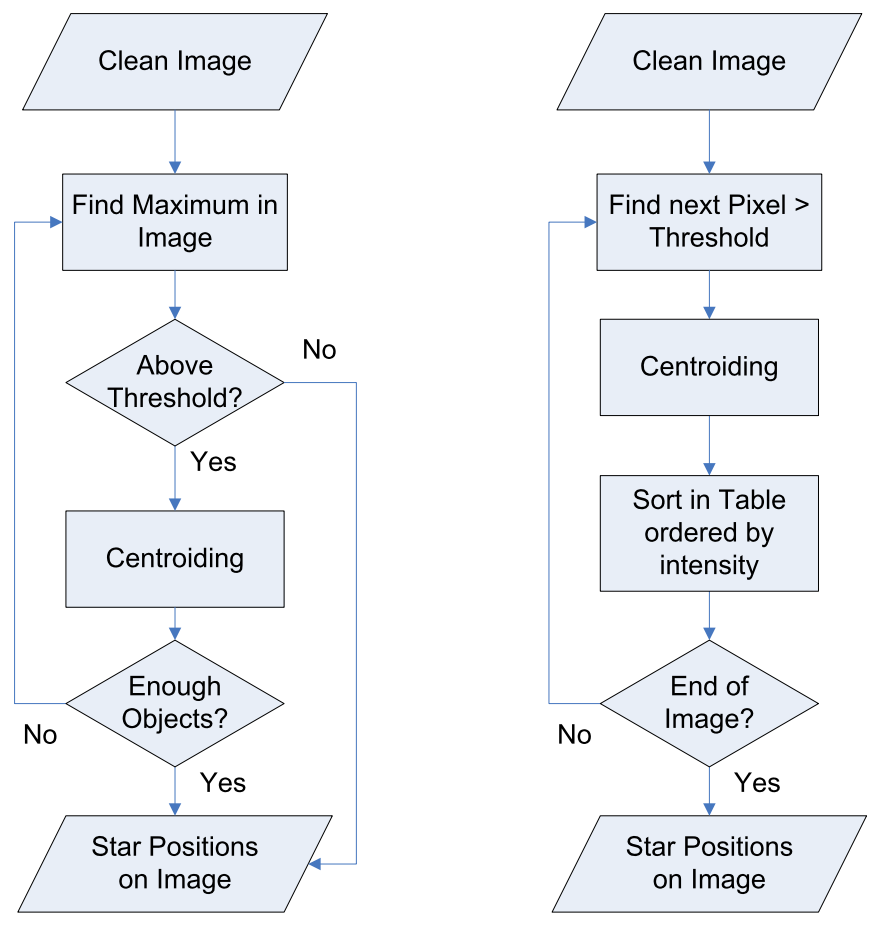

Fig. 3. Two different peak finding methods.

is it a good choice to count the saturated pixels in the image, because a very bright object such as a planet with a certain number of saturated pixels could be in the image. Then, it is counter productive to diminish the exposure time, fainter stars getting lost. Considering these arguments, it was chosen to set flagSaturated when more than one saturated pixels are present in at least four centroided objects. The control gets more robust by this measure.

The flag that is responsible for deciding upon the increase of the exposure time is called flagNoStars. This flag will be set if the centroiding delivers less than four stars. The star identification is then unable to be performed and the camera control block will augment the exposure time. A good value for the incrementation and decrementation step is $2 \mathrm{~ms}$. It allows both to have several different exposure time steps and to react rather quickly to changing conditions. The quick adaptation is necessary because the duration of the star tracker operating time will not be long.

\subsection{Image processing}

The image processing algorithm has been implemented using a recursive centroiding algorithm [9] together with a new idea for the CCD scanning part.

After the image subtraction avoiding the presence of bad pixels, two methods to select the centroids within the image are compared. Both of these peak finding methods are shown in Fig. 3. Several image processing techniques based on the peak localization are already presented as in Ref. [2].

The first method (Fig. 3 left side) consists of scanning the whole image every time, looking for the maximum grayscale value. Then, this value is compared to the threshold. If it is lower than the threshold, the whole centroiding process stops and the centroids calculated so far are transmitted to the star identification algorithm. If the intensity value surpasses the threshold, centroiding is performed on the concerned region. Afterwards, a check is performed to determine if the maximum number of objects fixed in the requirements is not exceeded. Depending on this check, the next object is searched or the centroids are forwarded to star identification.
The second (developed) method (Fig. 3 right side) only goes once through the image. As soon as it encounters a pixel with a value over the threshold, it performs centroiding of the object. Then, the object is sorted in a table (sorted by intensity), thereby making sure that only the brightest objects are transmitted to star ID and not only the first ones found. Once the whole image is scanned, the algorithm ends.

The advantage of the first algorithm is that the maximum number of objects processed is the one, which is fixed in the image processing assumption for the maximum number of stars. It also allows centroiding algorithms to most likely start in the center of the object, which is convenient for the normal point spread function. But the big drawback is that the whole image is scanned multiple times, that leads to longer processing times. The second algorithm has to deal with the fact that it may have to analyze many objects and always call a sorting algorithm, but at the end the processing time remains shorter. The average processing time using the maximum detection method is about $110 \mathrm{~ms}$ while in the sequential detection method is about $9.9 \mathrm{~ms}$ (note that the test has been performed on the actual star tracker computer, meaning with the $650 \mathrm{MHz}$ processor). This led to the choice of the sequential CCD scanning method.

\subsection{On-board star catalog}

Usually the on-board star catalog is camera-dependent. The selection of the catalog is an important task, as it gives the framework for a good functioning star identification. For the SHEFEX 2 mission, the Hipparcos star catalog will be used.

The catalog's cutoff magnitude is one important factor, but it is not enough to balance the other factor, the catalog uniformity. The developed star identification algorithm needs a minimum of 4 stars in each imaged frame just to have the ability to process the star identification. In reality, the distribution of the stars on the unit sphere in the ECI frame is far from being uniform. This means it is not always guaranteed to have enough stars in the FOV. To solve this problem, techniques to create a uniform star distribution have been developed [7]. Another possibility is to modify the cutoff magnitude in the region of interest. For the SHEFEX 2 mission, as the flight experiment is carried out in Australia, it was considered to develop an asymmetric star catalog. This would mean to take different cutoff magnitudes for each hemisphere, filling up the southern one and thinning out the northern one.

The creation of a uniform catalog is important in this mission. An advantage of this uniformity is also that some FOVs with a high number of stars lying close to each other is avoided. The asymmetric star distribution is shown in Fig. 4 and the uniform star distribution is shown in Fig. 5.

An inconvenience is though that to create a uniform distribution, one must allow lower stars magnitudes, meaning fainter stars, to be considered for the catalog. This requires a sensitive CCD with observable faint stars for the specified exposure time range. The asymmetric catalog doesn't have stars with magnitudes over 5.4 and only about 180 stars between 5.2 and 5.4, which represent the difference in stars between the hemispheres. The uniform catalog has about 450 stars in the range between 5.25 and 5.75 apparent magnitude. This means the star identification has to rely on faint stars in many cases.

The chosen catalog was a combination of both solutions, creating a uniform star catalog out of the asymmetric one integrating all stars with a magnitude up to 4.5 and filling up the remaining space with uniformly distributed stars from the asymmetric catalog. This led to a final result of 2023 stars which can be combined to 54804 reasonable star pair combinations to be used into the search algorithm. 


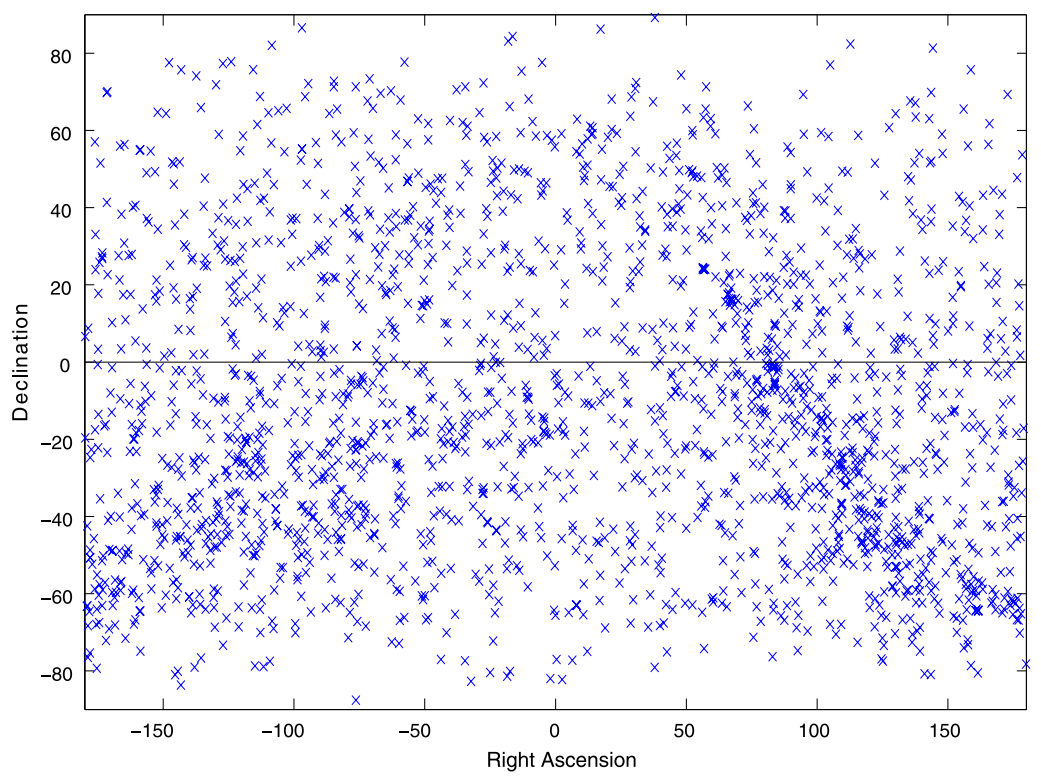

Fig. 4. Star distribution for the asymmetric catalog.

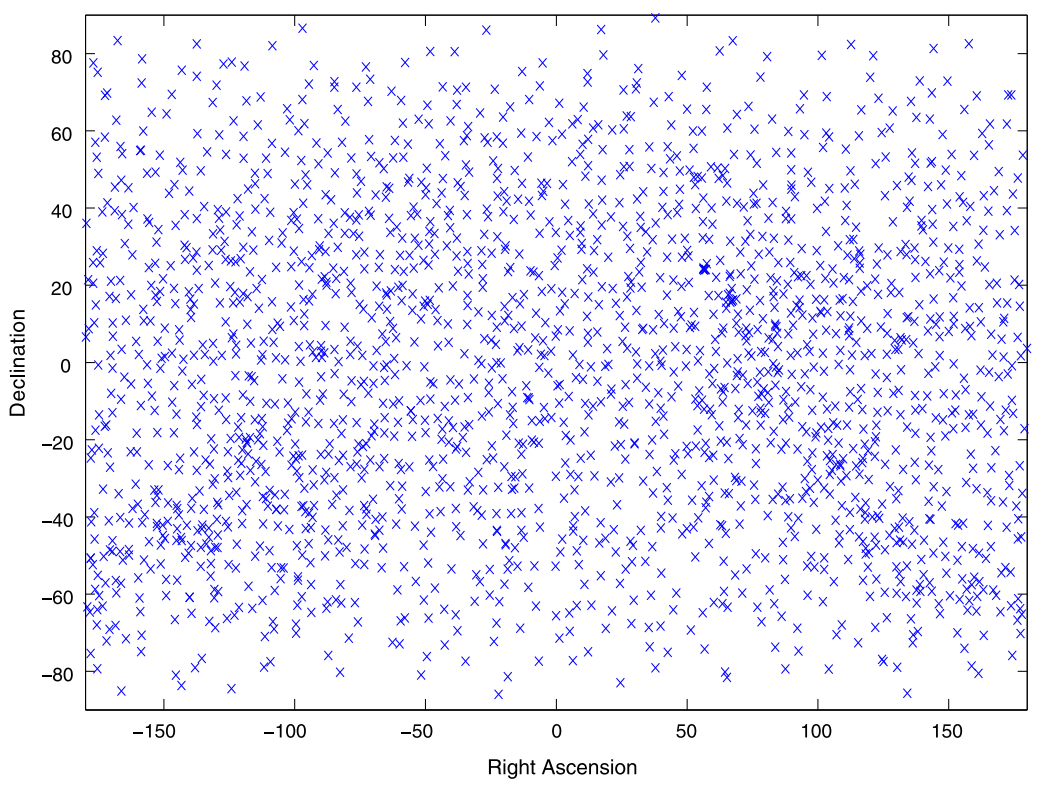

Fig. 5. Star distribution for the uniform catalog.

\subsection{Star identification}

Due to the expected small number of the imaged stars in the FOV, it was decided to use a geometrical algorithm with a database consisting of interstar angles. These interstar angles are chosen so that only those smaller than the maximum diagonal FOV size (here $18.57 \mathrm{deg}$ ) are taken into account. The so-calculated interstar angles are sorted and a $k$-Vector is created from them, in order to perform the fast $k$-Vector search [3]. The $k$-Vector table contains the index of both stars from each admissible star pair.

The general idea is based on star triangles and pyramids [5]. The first objective is to identify three stars arranged in a triangle. The next step is to try to identify another star, which would be the criterion for successful identification. Both steps require the database search with help of the $k$-Vector. To get an insight in the pattern search, it will be described in detail, so it can be used in the general flow chart as a black box. It does not matter in terms of identification process, if a triangle is to be found or a fourth star is trying to be added to the pattern to form four triangles; it will only affect the complexity.

Using the $k$-Vector search technique, the whole star identification algorithm is presented in Fig. 6. The input is an array with the centroids, therefore having a size corresponding to the fixed maximum number of stars times two ( $x$ - and $y$-value of the centroids). After having determined the real number of centroids in the table numStars $\leqslant$ maxNumStars, it is checked whether 4 or more centroids are available. In the negative case, the star ID is exited, returning a failure. The number of triangles that is corresponding to the number of centroids numStars could be calculated from

num $_{\text {triangles }}=\frac{\text { numStars! }}{(3 !)(\text { numStars }-3) !}$

First of all, a check is performed to be sure not to look for stars too close to each other within certain limit, because they cannot be 


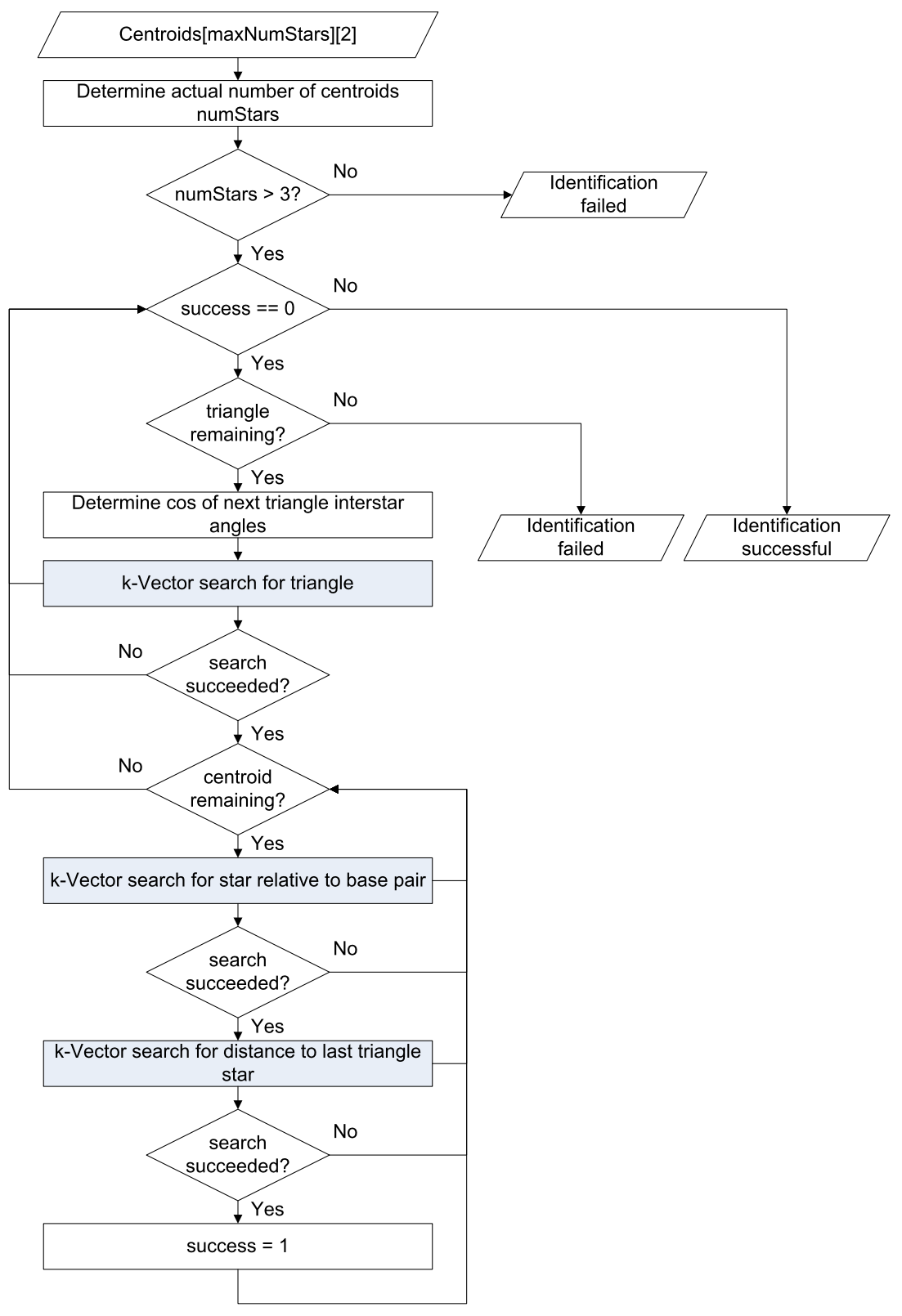

Fig. 6. Flow chart of the star identification algorithm.

distinguished by the centroiding. This limit value called cosLimit and is calculated by:

cosLimit $=q+m \cdot k_{\text {max }}-$ cosError

where $q$ is the $k$-Vector constant, $m$ is the slope of the $k$-Vector and $k_{\max }$ is the total length of the $k$-Vector [3]. The value cosError, that stands for the error tolerance given by centroiding errors, calibration errors and distortive optical effects. An approximate calculation of the cosError can be calculated by:

$$
\begin{aligned}
\cos \text { Error }= & c \cdot \mid \cos \left(F O V_{\text {max }}\right) \\
& -\cos \left(F O V_{\text {max }}-\text { centroidError } * \text { pixelSize } \frac{\mathrm{deg}}{\mathrm{pix}}\right) \mid
\end{aligned}
$$

In this approximation, $c$ is a constant factor that is determined by fine-tuning during tests. The centroidError is the centroiding error that could be set to a value between 0.1 and 0.3 pixel and multiplied by the angle per pixel, computed from the FOV and the CCD size (0.02 deg).

If the cosines determined by the algorithm don't fulfill the condition of being smaller than cosLimit, the $k$-Vector search signalizes an out-of-range problem and quits the block. If the $\operatorname{check}(\mathrm{s})$ were successful, a tolerance range for each of the cosines is computed. The range is delimited by a lower index and a higher index:

$$
\begin{aligned}
& i_{\text {low }}=\left\lfloor\frac{\cos \alpha-\cos \text { Error }}{m}-\frac{q}{m}\right\rfloor \\
& i_{\text {high }}=\left\lceil\frac{\cos \alpha-\cos \text { Error }}{m}+\frac{q}{m}\right\rceil
\end{aligned}
$$

where $\alpha$ is the angle between each of the measured star pairs. From the resulting list(s) of star pairs, a matching algorithm is supposed to find a triangle or a particular star combination.

The base triangle should be first identified. This is done via the $k$-Vector search for each star pair in this triangle. When a triangle (base) is correctly identified, a fourth star is taken from the 


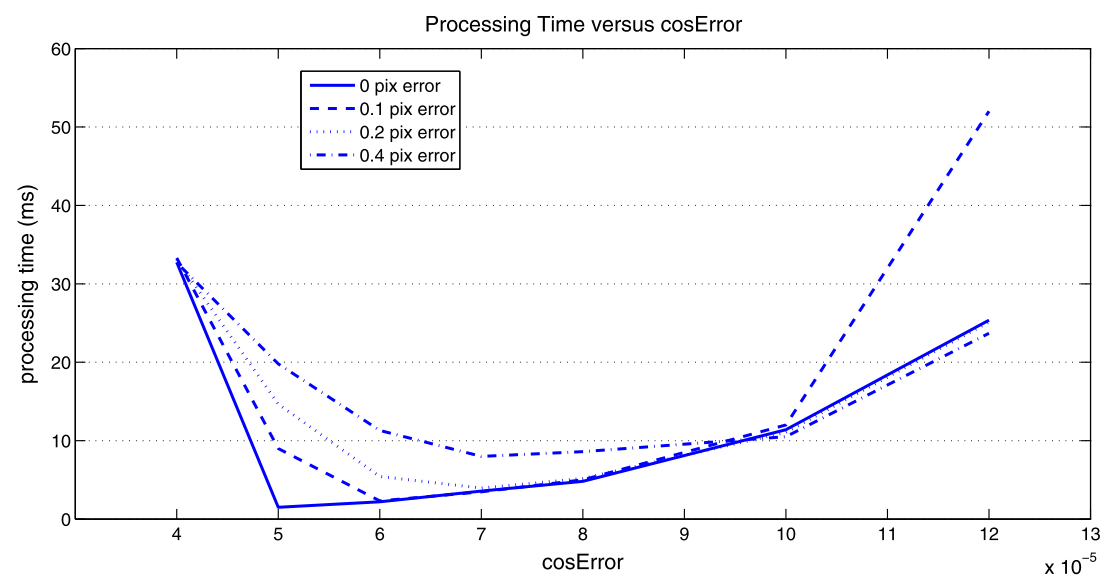

Fig. 7. Processing time with respect to cosError.

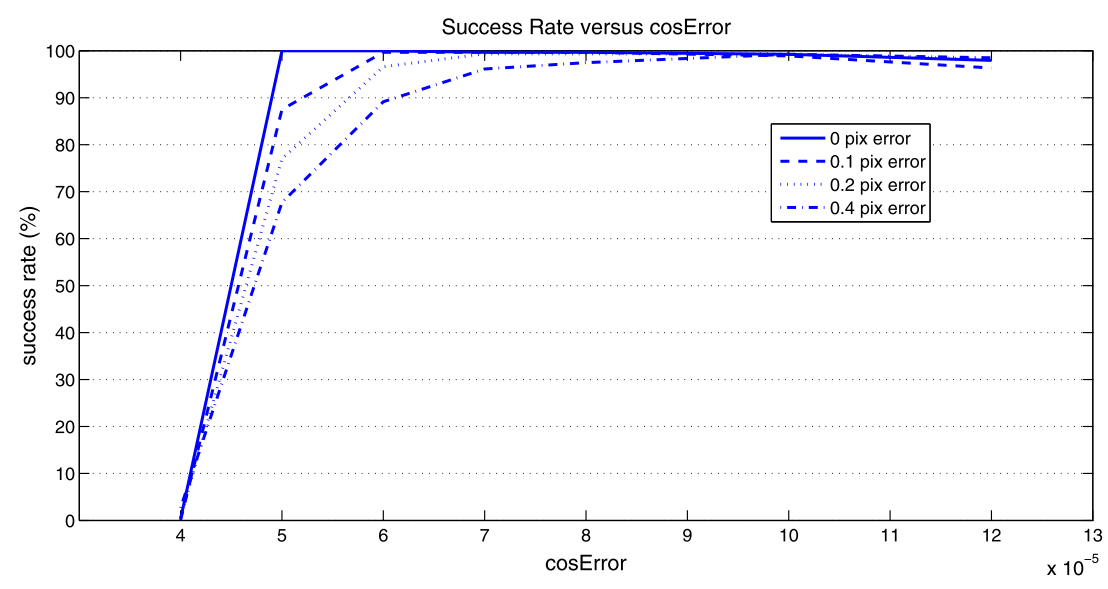

Fig. 8. Success rate with respect to cosError.

centroid array. The three interstar angles between this star and three base stars belonging to the identified triangle are computed and a star pair match is searched using the $k$-Vector search. If this search in turn is successful, it is already certain that a success will occur and the corresponding inertial and body vectors will be forwarded to the attitude determination. But, in order to gain precision, the remaining centroids of the array are also tried to be identified in the same manner as was the fourth star (using the same base triangle).

It can also be observed that if the fourth star does not match with the triangle, the fifth in the array (if available) is checked and so on. This makes the algorithm more robust in terms of nonstellar objects (spikes). The last step not figuring in the chart in Fig. 6 is that, in case of success, the body vectors are stored in an array and the corresponding inertial vectors are taken from the on-board catalog and stored in the same order in a second array. Both of them will be used for attitude determination.

In general, it can already be observed that the processing time of the star ID algorithm is highly dependent on the position of the stars in the centroid array, on the number of spikes within the image, on the tolerance error of the star ID and on the question if more than three stars figuring in the catalog are within the array. This aspect will be investigated in the tests.

Once the star identification algorithm is successfully processed, all the imaged star vectors and the corresponding cataloged vectors are then forwarded to the attitude determination algorithm. The fast estimation algorithm ESOQ 2 [4] is utilized for the attitude determination without modification as it gives the most accurate and reliable attitude estimation.

\subsubsection{Star identification parameters}

A simulated test environment is created to test the star identification algorithm. As input, random attitudes were generated and the stars within each FOV are selected. Additionally, spikes (nonstar objects) could be added in random percentages. The centroids of all objects were determined and given as input to the star ID. The whole centroiding process and digitization errors which are represented by a total image processing error that are added to the centroids.

The parameters that were varied during the tests were the number of spikes in the image and the error tolerance, namely the parameter cosError (defined in Eq. (3)). At the output, the observed values were the number of successful identifications, the processing time and the number of wrong identifications.

The first test was carried out on the target computer and the goal was to determine the variation of the processing time with respect to the cosError parameter for different added centroiding errors. The corresponding graph is shown in Fig. 7. It was realized using 1000 random attitudes and a maximum number of 15 centroids. All the curves show a minimum processing time. This can be explained by the fact that for low values of cosError, the star ID is seldom successful and therefore, a big number of triangles is scanned through each time. On the other hand, if cosError gets too large, the number of interstar angle candidates for one star pair gets also large and it takes longer to match the pairs to patterns. Another observation is that for higher centroiding errors, the optimum moves to the right, because the searched interstar angles cannot be found in a narrow range anymore. 
M. Samaan, S. Theil / Aerospace Science and Technology $\bullet \bullet \bullet(\bullet \bullet \bullet \bullet) \bullet \bullet \bullet-\bullet \bullet$

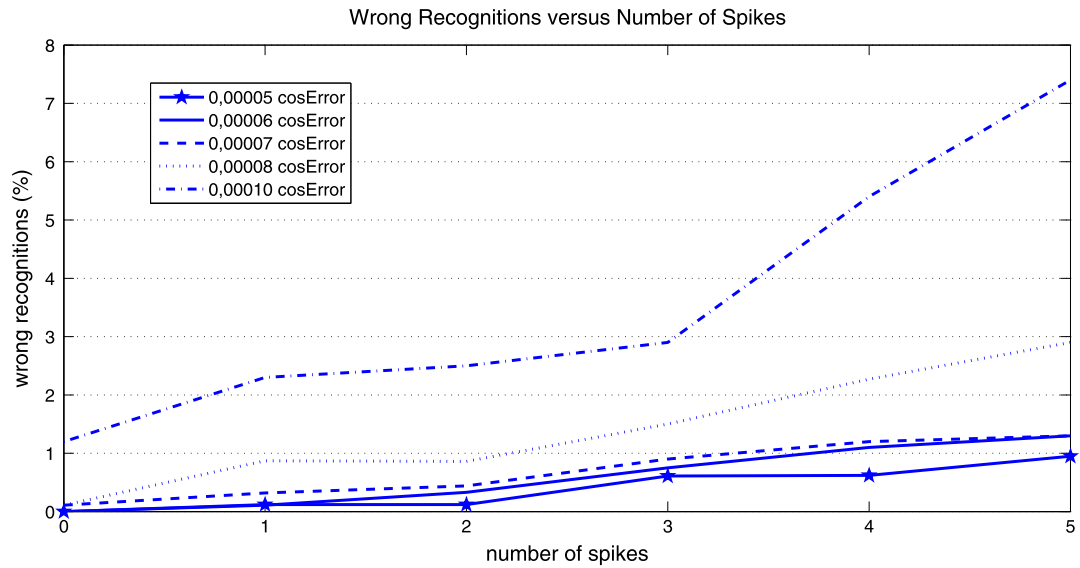

Fig. 9. Wrong recognition rate with respect to number of spikes.

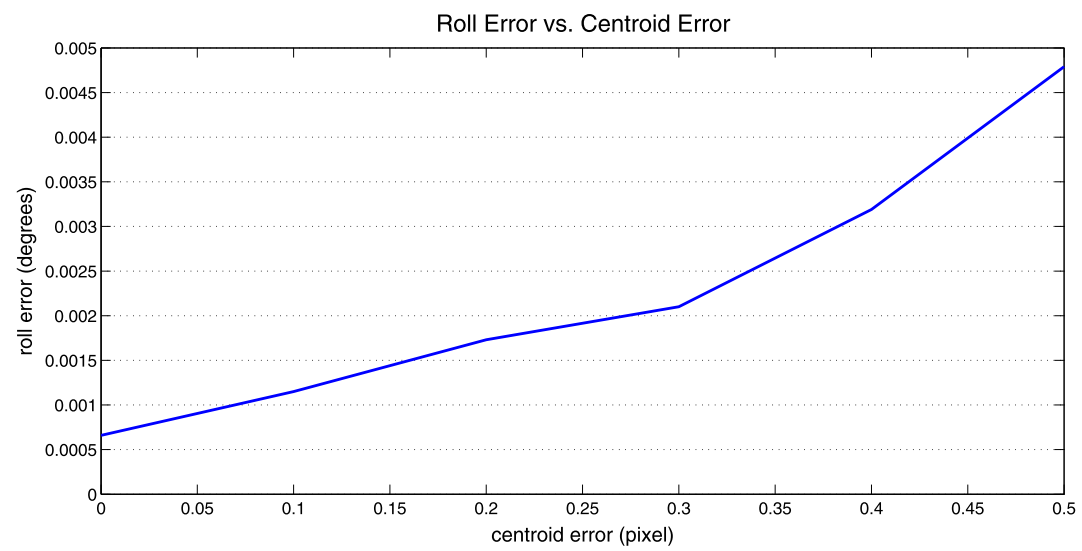

Pitch Error vs. Centroid Error

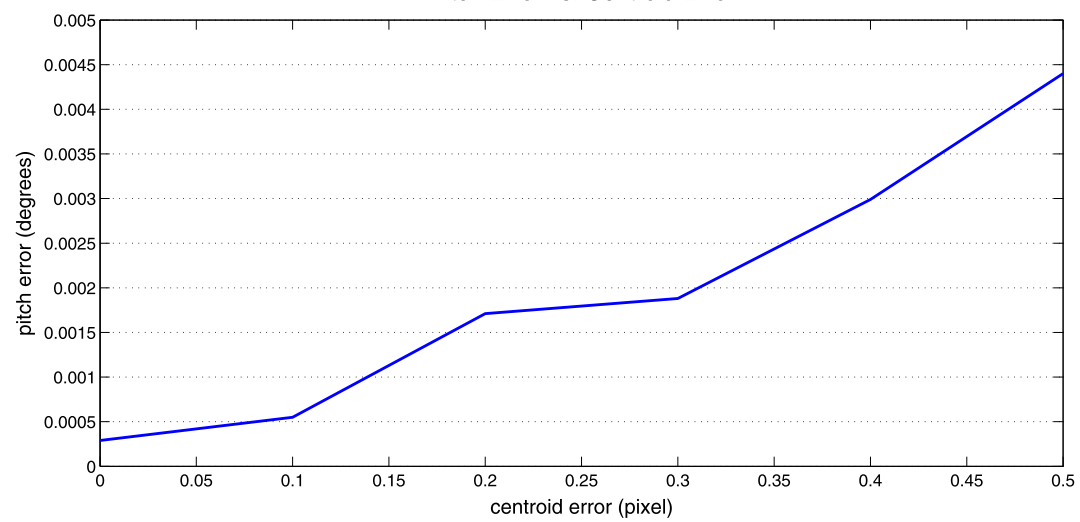

Yaw Error vs. Centroid Error

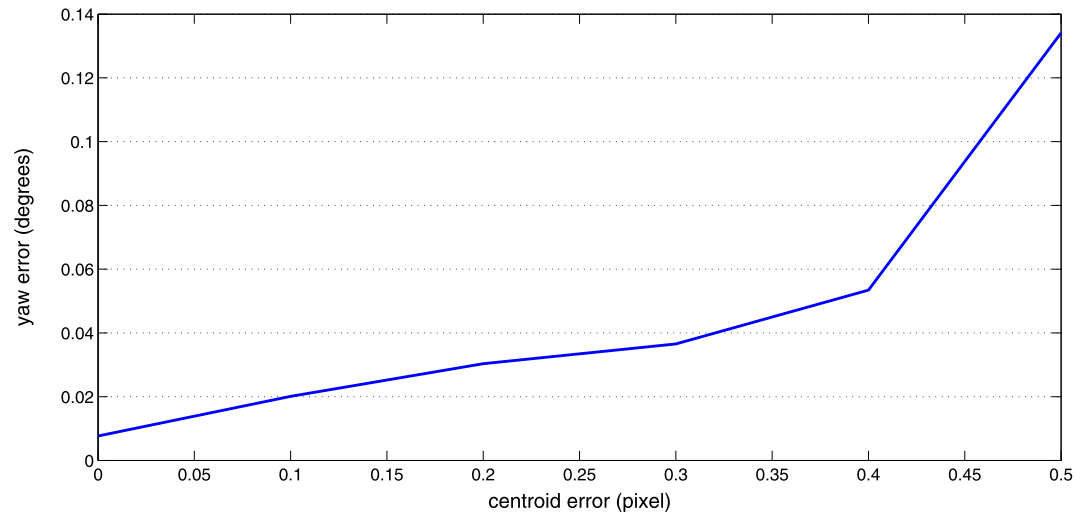

Fig. 10. Roll, pitch, yaw error with respect to the centroiding error. 
The second parameter on which the tests were focused, was the success rate seen in Fig. 8. The curve is rising with rising cosError. The probability to find the pattern within a larger range of possibilities is more probable. It is also obvious that the smaller the centroiding error, the higher the success rate.

In a last test, the wrong recognition rate is determined (Fig. 9), depending on the number of spikes in the "image". The first effect seen is that when more spikes are introduced, the number of wrong recognitions rises. Furthermore, when the cosError value is increased, the wrong recognition rate also increases. This test also used 1000 random attitudes. The bottom line of the test series is that a trade off between the different factors has to be found, limiting the wrong identification rate as much as possible while still having a good success rate and a reasonable speed. Knowing that centroiding errors of 0.1 to 0.2 pixel are expected, the choice is to use a cosError value between 0.00005 and 0.00006 , thereby also keeping the wrong identification rate at a low level.

The angular errors in terms of roll, pitch and yaw error was determined using a series of tests with 1000 random attitudes and applied centroiding error on each of the stars within the FOV. The star identification and attitude determination were then carried out and the rotation errors calculated. The total attitude errors results are shown in Fig. 10. The first look reveals steadily increasing errors with increasing centroid errors. This is easy to understand as the estimation gets worse when the centroids are less accurate.

The requirements state that the error around all three axes cannot exceed 0.1 degrees. By taking a look at the test results, it can be seen that this condition is not met for centroiding errors greater than 0.45 pixel. In that case, the errors for roll and pitch are still far from being critical, but the yaw error surpasses 0.1 degrees. This phenomenon of the yaw error (meaning the error around the optical axis, which normally is the $z$-axis) being larger by one or two orders of magnitude is common for star sensors. It arises from the two dimensional structure of the CCD. Indeed, around the $x$ - and $y$-axis, the accuracy is determined by the angular size of a pixel, being around 0.02 degrees in this application. Around the optical axis, in the best case, when the star lies close to the CCD border, the obtained resolution ranges around 0.2 degrees (for $25 \mathrm{~mm}$ focal length). The closer the star is to the optical axis, the worse the accuracy in terms of yaw angle. In the SHEFEX 2 mission, this inconvenience has to be accepted.

\section{Night sky tests}

In order to test the whole processing chain (End-to-End) using the engineering module hardware, several night sky tests were carried out.

As the first step in these tests, the focusing for the camera is adjusted in order to find a suitable working focusing range. Based on the successful attitude obtained from the image processing programs, the focusing range is adjusted and recorded, which found to be $\pm 0.1 \mathrm{~mm}$ from the sharp focus point.

Moreover, the camera parameters, the focal length and the principle point offsets were then calibrated using different calibration algorithms [8]. The End-to-End image processing algorithms were successfully tested for different scenarios. The camera calibrated focal length was found equal to $24.95 \mathrm{~mm}$. Although one of the night tests was done during a full Moon sky, we noticed that the sensor is fully functional, which is one of the worst case scenarios.

The integration time is automatically controllable using the conditions described before. It is found that 35 to $50 \mathrm{~ms}$ is a good working range for the integration time. Thousands of star images were captured and instantaneously processed showing different orientations, different integration times, different camera focusing, and different degrees of image smear. They were processed using the uniform Hipparcos catalog. The End-to-End success rate for the

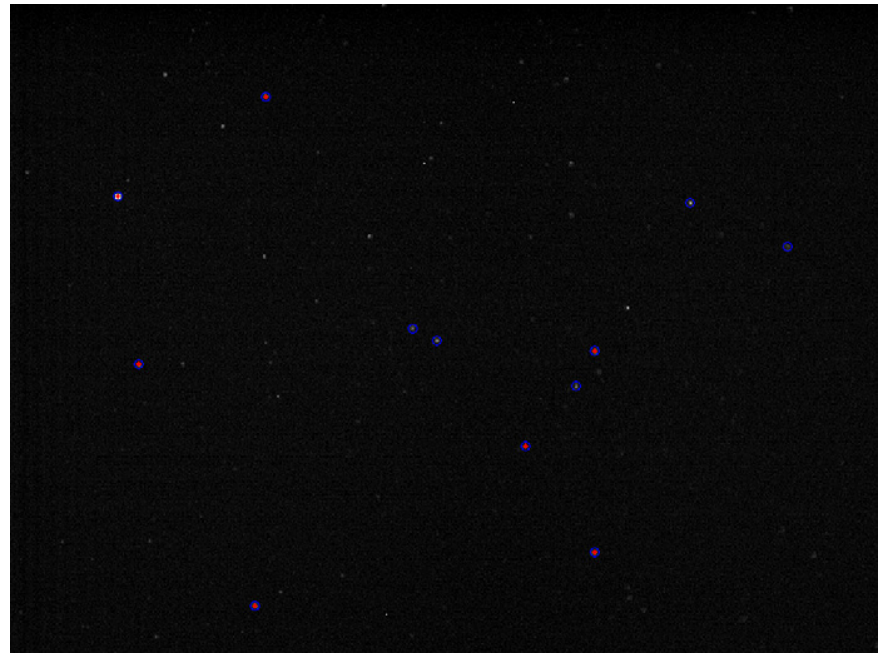

Fig. 11. Night sky image with the centroided stars in blue and the identified stars in red. (For interpretation of the references to color in this figure, the reader is referred to the web version of this article.)

on-ground night sky test was more than $90 \%$. The night sky tests proved the functionality of all the algorithms. On the other hand, the camera and software parameters were adjusted and fixed for the flight module.

One of the images test results (using $40 \mathrm{~ms}$ integration time) is presented in Fig. 11. The centroided stars (in this image were 12) are shown in blue while the identified stars are shown in red.

Furthermore, many post processing tests using the captured night sky images were later processed, for different values of cosError and focal length. Many images still could already be identified with the slightly changed camera and algorithms parameters. Keeping in mind the discussion about processing speed in the last section, a value of 0.000055 is a good choice cosError. This choice is reasonable, especially considering that a closer look at the analyzed images reveals that some of them show significant image smear. Most off all the images with smear of corresponding to angular rates of $2 \mathrm{deg} / \mathrm{s}$ or less where successful identified. However, for angular rates greater than $4 \mathrm{deg} / \mathrm{s}$ the stars could be rarely identified.

\section{Conclusion}

The achievable objective of this work was to develop and implement a software processing chain together with a camera control unit coupling the software and the star tracker hardware. The designed and integrated star sensor will be used as a primary attitude determination sensor in the SHEFEX 2 mission. Each software module was written separately and tested on its own before being integrated into the chain. The modules proved to be robust against unwanted conditions and as accurate as required. They are based on existing concepts, but also include new algorithms and ideas for image processing.

The complete chain has been tested both with simulated images and during night sky tests, where it revealed more than $97 \%$ star identification success rate and also proved the robustness regarding non-catalog objects, noise and small angular rates.

The next step towards the integration in the INS is the creation of a data handling interface. This means that the simple outputs of the quaternion, health flags, etc. must be replaced by commands and binary information in order to enable automatic interaction with the navigation computer. After this development, the whole INS can be tested in the loop. 


\section{References}

[1] http://en.wikipedia.org/wiki/PC/104.

[2] C. Liebe, Accuracy performance of star trackers - a tutorial, Journal of IEEE, Transaction of Aerospace and Electronic Systems 38 (2) (April 2002) 587599.

[3] D. Mortari, Search-less algorithm for star pattern recognition, Journal of the Astronautical Sciences 45 (2) (1997) 179-194.

[4] D. Mortari, Second estimator of the optimal quaternion, Journal of Guidance, Control, and Dynamics 23 (5) (2000) 885-888.

[5] D. Mortari, M. Samaan, J. Junkins, Lost-in-space pyramid algorithm for robust star pattern recognition, in: Guidance and Control Conference, Breckenridge, CO, January-February 2001, Paper AAS 01-004.

[6] Prosilica Inc., User Manual EC655, 2005.

[7] M. Samaan, C. Bruccoleri, D. Mortari, J.L. Junkins, Novel techniques for the creation of a Uniform Star Catalog, in: AAS/AIAA Astrodynamics Specialist Conference, August 2003, Paper AAS 03-609.

[8] M. Samaan, T. Griffith, J. Junkins, Autonomous on-orbit calibration of star trackers, in: Core Technologies for Space Systems Conference, Colorado Springs, CO, 27-30 November 2001, AAS 01-325.

[9] M. Samaan, D. Mortari, J.L. Junkins, Recursive mode star identification algorithms, Journal of IEEE, Transaction of Aerospace and Electronic Systems 41 (4) (October 2005) 1246-1254.

[10] S. Theil, M. Schlotterer, M. Hallmann, Hybrid navigation system for the SHEFEX2 mission, in: Proceedings of the AIAA Guidance, Navigation and Control Conference and Exhibit 2008, August 2008, No. AIAA 2008-6991.

[11] S. Theil, S. Steffes, M. Samaan, Hybrid navigation system for space planes, launch and re-entry vehicles, in: 16th AIAA/DLR/DGLR International Space Planes and Hypersonic Systems and Technologies Conference, Bremen, Germany, 19-22 October 2009, AIAA 2009-7381. 